\section{(1)}

CrossMark

\title{
Cardiometabolic correlates of sleep disordered breathing in Andean highlanders
}

\author{
Luu V. Pham (10) ${ }^{1}$, Catherine H. Miele ${ }^{1}$, Noah G. Schwartz² ${ }^{2}$ Rafael S. Arias ${ }^{3}$, \\ Adi Rattner ${ }^{3}$, Robert H. Gilman ${ }^{4}$, J. Jaime Miranda $\mathbb{1}^{5,6}$, Vsevolod Y. Polotsky ${ }^{1}$, \\ William Checkley ${ }^{1,4}$ and Alan R. Schwartz ${ }^{1}$
}

Affiliations: ${ }^{1}$ Division of Pulmonary and Critical Care and Sleep Medicine, Dept of Medicine, School of Medicine, Johns Hopkins University, Baltimore, MD, USA. ${ }^{2}$ University of Chicago Pritzker School of Medicine, Chicago, IL, USA. ${ }^{3}$ School of Medicine, Johns Hopkins University, Baltimore, MD, USA. ${ }^{4}$ Program in Global Disease, Epidemiology and Control, Dept of International Health, Bloomberg School of Public Health, Johns Hopkins University, Baltimore, MD, USA. ${ }^{5}$ CRONICAS Center of Excellence in Chronic Diseases, Universidad Peruana Cayetano Heredia, Lima, Peru. ${ }^{6}$ School of Medicine, Universidad Peruana Cayetano Heredia, Lima, Peru.

Correspondence: Luu Pham, Johns Hopkins Sleep Disorders Center, Asthma and Allergy, 4th Floor, 5501 Hopkins Bayview Circle, Baltimore, MD 21224, USA. E-mail: Ipham1 Qjhmi.edu

@ERSpublications

In highlanders, nocturnal hypoxaemia and sleep apnoea were associated with differential outcomes http://ow.ly/1k6z309MyDR

Cite this article as: Pham LV, Miele $\mathrm{CH}$, Schwartz NG, et al. Cardiometabolic correlates of sleep disordered breathing in Andean highlanders. Eur Respir J 2017; 49: 1601705 [https://doi.org/10.1183/ 13993003.01705-2016].

ABSTRACT Associations between sleep disordered breathing (SDB) and cardiometabolic outcomes have not been examined in highlanders.

We performed nocturnal polygraphy in Peruvian highlanders $(3825 \mathrm{~m})$. Multivariable linear regression models examined associations between SDB metrics and haemoglobin, glucose tolerance (haemoglobin A1c (HbAlc)), fasting glucose, homeostatic model-based assessments of insulin resistance and $\beta$-cell function (HOMA-IR and HOMA- $\beta$, respectively), blood pressure, and lipids, while adjusting for age, sex, body mass index (BMI) and wake oxygenation.

Participants ( $\mathrm{n}=187 ; 91$ men) were (median (interquartile range)) 52 (45-62) years old, and had a BMI of 27.0 (24.3-29.5) $\mathrm{kg} \cdot \mathrm{m}^{-2}$ and $87 \%$ (85-88\%) oxyhaemoglobin (arterial oxygen) saturation during wakefulness. In fully adjusted models, worsening nocturnal hypoxaemia was associated with haemoglobin elevations in men $(\mathrm{p}=0.03)$, independent of wake oxygenation and apnoea-hypopnoea index (AHI), whereas worsening wake oxygenation was associated with haemoglobin elevations in older women $(p=0.02)$. In contrast, AHI was independently associated with HbA1c elevations $(p<0.05)$. In singlevariable models, nocturnal hypoxaemia was associated with higher HbA1c, HOMA-IR and HOMA- $\beta$ $(\mathrm{p}<0.001, \mathrm{p}=0.02$ and $\mathrm{p}=0.04$, respectively), whereas AHI was associated with HOMA-IR, systolic blood pressure and triglyceride elevations $(\mathrm{p}=0.02, \mathrm{p}=0.01$ and $\mathrm{p}<0.01$, respectively). These associations were not significant in fully adjusted models.

In highlanders, nocturnal hypoxaemia and sleep apnoea were associated with distinct cardiometabolic outcomes, conferring differential risk for excessive erythrocytosis and glucose intolerance, respectively. 


\section{Introduction}

Globally, approximately 150 million people live at altitudes $>2500 \mathrm{~m}$. Ambient hypoxia makes highlanders chronically hypoxaemic, which further worsens during sleep. Hypoxia causes increases in ventilatory chemosensitivity, which predisposes to central sleep apnoea with intermittent nocturnal oxyhaemoglobin desaturations. Ambient hypoxia further amplifies sleep-related decreases and swings in nocturnal oxyhaemoglobin saturation since oxygenation lies on the steep rather than flat portion of the oxyhaemoglobin dissociation curve. Thus, altitude may represent a "perfect storm" for sleep disordered breathing (SDB), exposing highlanders to uncommon degrees of sleep apnoea and nocturnal oxyhaemoglobin desaturation, and related cardiometabolic sequelae [1]. Nevertheless, the effects of chronic and intermittent nocturnal hypoxaemia have not been well characterised in highlanders.

It is well recognised that chronic nocturnal hypoxaemia is associated with pulmonary hypertension, cor pulmonale and mortality. In patients with severe chronic obstructive pulmonary disease, nocturnal hypoxaemia is a predictor of pulmonary hypertension and mortality [2]. Oxygen treatment trials in patients with sustained nocturnal hypoxaemia have, however, yielded equivocal haemodynamic responses and no improvements in mortality [3-6]. In morbid obesity, chronic nocturnal hypoxaemia has also been associated with elevations in pulmonary artery pressure $[7,8]$, secondary polycythaemia and frank cor pulmonale [9]. Nevertheless, clinical comorbidities in these patients may confound the effects of chronic nocturnal hypoxaemia on pulmonary haemodynamics and overall morbidity and mortality. At high altitude, a similar constellation of clinical findings can develop in normal highlanders, without comorbid obesity or lung disease [10-12].

In contrast to chronically hypoxaemic individuals, patients with intermittent hypoxaemia from sleep apnoea exhibit a different spectrum of clinical comorbidities. Specifically, sleep apnoea has been primarily associated with systemic rather than pulmonary hypertension and left heart dysfunction, including hypertension, coronary artery disease, heart failure and cardiovascular mortality [13-19]. Sleep apnoea has also been associated with metabolic dysfunction, including glucose intolerance, hyperlipidaemia and fatty liver disease [20-23]. While treating sleep apnoea can mitigate these cardiometabolic sequelae [24, 25], it is still likely that concomitant obesity, a potent sleep apnoea risk factor in lowland populations, can confound these associations.

The purpose of this study was to examine the cross-sectional relationships between specific SDB patterns and cardiometabolic outcomes in highlanders. Recently, we have found unique associations between cardiometabolic outcomes, sleep-related breathing disturbances [26] and hypoxaemia during wakefulness in Peruvian highlanders [27], suggesting a pathogenic role for SDB. We hypothesised that chronic and intermittent nocturnal hypoxaemia are differentially associated with specific biomarkers of cardiometabolic dysfunction, i.e. haemoglobin concentration and glucose levels, respectively. We addressed this hypothesis by performing nocturnal assessments of SDB in highland participants from the CRONICAS cohort study, who were well characterised for a range of cardiometabolic biomarkers [28].

\section{Methods}

\section{Study setting and participant selection}

The present study is an ancillary study of the CRONICAS cohort study, an epidemiological study designed to characterise risk factors for noncommunicable chronic diseases in four distinct settings differing in altitude, air quality and urbanisation [28]. A convenience sample of highlanders aged 35-75 years was recruited from the high-altitude site (Puno, Peru; $3825 \mathrm{~m}$ ). These subjects were long-standing native highlanders of Aymara and Quechua descent. The majority of subjects (80\%) were urban residents of Puno. In those for whom occupation data was available, government or private sector was the largest source of employment (39\%), followed by agriculture (14\%). As part of this study, demographic, anthropometric measurements and biomarkers of chronic disease were recorded. The study protocol was approved by the Institutional Review Boards of the Johns Hopkins Bloomberg School of Public Health (Baltimore, MD, USA) and Universidad Peruana Cayetano Heredia and A.B. PRISMA (Lima, Peru). All participants provided verbal informed assent because of low literacy rates.

Support statement: This project was funded with federal funds from the US National Heart, Lung and Blood Institute (NHLBI), National Institutes of Health (NIH), Dept of Health and Human Services, under contract HHSN268200900033C. L. Pham was further supported by a NIH National Research Service Award (5T32HL1109523). J. Jaime Miranda is supported by the Fogarty International Centre (R21TW009982), Grand Challenges Canada (0335-04), International Development Research Center Canada (106887-001), Inter-American Institute for Global Change Research (IAI CRN3036), Medical Research Council UK (M007405), NHLBI (U01HL114180), and National Institutes of Mental Health (U19MH098780). V.Y. Polotsky was supported by NHLBI grants (R01s HL133100, HL128970 and HL080105) and by the National Institute of Environmental Health Sciences P50ES018176. A.R. Schwartz was supported by NHLBI grants (HL128970 and HL133100). ResMed Ltd provided ApneaLink recording devices. Funding information for this article has been deposited with the Crossref Funder Registry.

Conflict of interest: Disclosures can be found alongside this article at erj.ersjournals.com 
Daytime clinical assessment and laboratory analyses

Methods for clinical assessments and laboratory analyses were described previously [28]. Briefly, trained fieldwork personnel obtained anthropometrics and vital signs in triplicate using standardised techniques. Blood pressure was measured in triplicate using an automatic monitor. Venous blood samples were obtained after fasting between 8 and $12 \mathrm{~h}$. Plasma glucose was measured using an enzymatic colorimetric method, serum insulin using electrochemiluminescence and haemoglobin A1c (HbA1c) using high-performance liquid chromatography. All samples were analysed in a single facility. Quality assurance was performed with regular external standards and internal duplicate assays.

\section{Nocturnal recording methods}

Study subjects underwent nocturnal polygraphy with continuous recordings of nasal flow, respiratory effort and pulse oximetry (ApneaLink Plus; ResMed, Bella Vista, New South Wales, Australia). Simultaneous wrist actigraphy (Actiwatch 16 or 64; Philips Respironics, Amsterdam, The Netherlands) provided an indicator of sleep/wake state and movement arousals [29]. Study participants were instructed to start recordings at bedtime and stop at final awakening. A minimum of $4 \mathrm{~h}$ of recording was required; studies of $<4 \mathrm{~h}$ were repeated.

\section{Analysis \\ Independent variables}

We characterised SDB by the frequency of apnoeas and hypopnoeas (apnoea-hypopnoea index (AHI)) in accordance with the 2007 American Academy of Sleep Medicine recommended criteria [30]. Specifically, apnoeas were scored when there was absence of flow for $\geqslant 10$ s. Hypopnoeas were scored when a $>30 \%$ reduction in flow resulted in $\geqslant 4 \%$ desaturation or movement arousal.

The mean nocturnal arterial oxygen saturation measured by pulse oximetry $\left(\mathrm{SpO}_{2}\right)$ was chosen to represent nocturnal hypoxaemia severity and was calculated as the average $\mathrm{SpO}_{2}$ during sleep epochs. The mean nocturnal $\mathrm{SpO}_{2}$ among subjects was normally distributed, forming a continuum. Although we also sought to determine whether meaningful $\mathrm{SpO}_{2}$ thresholds could be established (e.g. percentage of sleep time with $\mathrm{SpO}_{2}<90 \%$, etc.) to quantify nocturnal hypoxaemic stress, we found that the distributions of these metrics varied widely with minimal changes in the threshold and were not normally distributed. Thus, time spent below a given $\mathrm{SpO}_{2}$ was unlikely to yield a readily interpretable predictor of cardiometabolic outcomes. We therefore chose mean $\mathrm{SpO}_{2}$ as an indicator of nocturnal hypoxaemia in highlanders.

In modelling cardiometabolic outcomes, we recognised that the severity of nocturnal hypoxaemia could be affected by levels of daytime hypoxaemia and apnoeic episodes. To account for these factors, we further considered two nocturnal $\mathrm{SpO}_{2}$ components: 1) $\mathrm{SpO}_{2}$ during wakefulness, which was measured prior to sleep onset at the start of the recording, and 2) the degree to which $\mathrm{SpO}_{2}$ deviated from expected levels during sleep after accounting for daytime hypoxaemia and frequency of apnoeic events (i.e. $\mathrm{SpO}_{2}$ residuals). These $\mathrm{SpO}_{2}$ residuals reflected the severity of nocturnal hypoxaemia (the more negative the $\mathrm{SpO}_{2}$ residual, the greater the severity of nocturnal hypoxaemia), independent of daytime $\mathrm{SpO}_{2}$ and apnoeic activity.

\section{Outcomes}

Our primary outcomes were haemoglobin, a marker of chronic mountain sickness (CMS) [31], and the circulating components of the metabolic syndrome. Specifically, we examined haemoglobin, HbA1c, fasting glucose, homeostatic model-based assessments of insulin resistance and $\beta$-cell function (HOMA-IR and HOMA- $\beta$, respectively), blood pressure, and triglyceride and high-density lipoprotein cholesterol as continuous outcomes. HOMA- $\beta$ and HOMA-IR were calculated from fasting glucose and insulin levels using previously described methods [32]. Excessive erythrocytosis was defined as haemoglobin $>21 \mathrm{~g} \cdot \mathrm{dL}^{-1}$ in men and $>19 \mathrm{~g} \cdot \mathrm{dL}^{-1}$ in women.

\section{Statistics}

Analyses were performed with R (www.r-project.org). Linear regression models were built in three stages. First, single-variable models examined biomarkers as functions of nocturnal $\mathrm{SpO}_{2}$ and AHI. In these models, biomarkers with a skewed distribution were normalised with Box-Cox transformations. In addition, we censored test subjects who had a diagnosis of or were actively treated for cardiometabolic diseases (e.g. diabetes, hypertension) from analysis. Sensitivity analyses were performed to confirm that the exclusion of these subjects did not significantly influence model results.

In the second stage, multivariable models were developed to examine the independent effects of SDB while adjusting for $\mathrm{SpO}_{2}$ during wakefulness. During this stage, $\mathrm{SDB}$ was modelled by $\mathrm{AHI}$ and the degree to which $\mathrm{SpO}_{2}$ deviated from expected levels after accounting for AHI and $\mathrm{SpO}_{2}$ during wakefulness $S_{\mathrm{pO}_{2}}$ residuals. In the final stage, the multivariable models were extended to adjust for potential influences of 
age, sex and obesity. To account for potential effects of menses and menopause on haemoglobin in women, analyses were stratified by sex and age $(\geqslant 55$ or $<55$ years old).

Our sample size was based on the number of observations required to attribute a significant amount of the variance $\left(r^{2}\right)$ in haemoglobin to differences in nocturnal hypoxaemia. As haemoglobin concentration is a continuous measure and was modelled as a function of multiple variables with linear regression, we examined the variance in this outcome that could be attributed to nocturnal hypoxaemia and potential confounding variables (e.g. BMI, sex and age). Within this framework, we calculated that a sample size of 160 subjects would enable the detection of a partial $r^{2}$ of $>0.05$ related to the nocturnal hypoxaemia with $>80 \%$ power while assuming a false type I error rate of 0.05 . To account for potential loss of data in up to $20 \%$ of test subjects, we sought to enrol 200 participants. Statistical inferences were drawn for factors achieving a $\mathrm{p} \leqslant 0.05$ level of significance.

\section{Results}

\section{Subject characteristics}

Study subject characteristics are presented in table 1. In total, 187 subjects were included, who were evenly split between men and women. In median, they were middle aged and mildly overweight but not obese. During wakefulness, highlanders had $\mathrm{SpO}_{2}$ percentage values in the high 80s, which decreased to the low 80s during sleep. Sleep apnoea was highly prevalent, with $>75 \%$ of highlanders demonstrating sleep apnoea (AHI $\geqslant 5$ events $\cdot h^{-1}$ ) characterised by a mix of central and obstructive events. Apnoeic events were often associated with dips in $\mathrm{SpO}_{2}$ percentage values into the 70s. Arousals from sleep, as detected by actigraphy, were infrequent and occurred a median of 5.6 events $\cdot \mathrm{h}^{-1}$. While apnoeic episodes contributed

\section{TABLE 1 Subject characteristics}

\begin{tabular}{|c|c|}
\hline Subjects & 187 \\
\hline Male/female & $91 / 96$ \\
\hline Age years & $52(45-62)$ \\
\hline \multicolumn{2}{|l|}{ Anthropometrics } \\
\hline Body mass index $\mathrm{kg} \cdot \mathrm{m}^{-2}$ & $27.0(24.3-29.5)$ \\
\hline Waist circumference $\mathrm{cm}$ & $90.0(82.2-98.5)$ \\
\hline Hip circumference cm & $95.5(90.8-99.4)$ \\
\hline Waist-to-hip ratio & $0.9(0.9-1.0)$ \\
\hline \multicolumn{2}{|l|}{ Oxygenation } \\
\hline $\mathrm{SpO}_{2}$ during wakefulness $\%$ & $87(85-88)$ \\
\hline Mean nocturnal $\mathrm{SpO}_{2} \%$ & $83.3(81.7-84.7)$ \\
\hline Mean nadir $\mathrm{SpO}_{2}$ after apnoeic events \% & $79.8(77.9-81.5)$ \\
\hline \multicolumn{2}{|l|}{ Sleep characteristics } \\
\hline Total recording time min & $436.5(381.4-376.4)$ \\
\hline Total sleep time min & $401.2(349.0-439.0)$ \\
\hline Arousal index events $\cdot \mathrm{h}^{-1}$ & $5.6(3.9-7.9)$ \\
\hline \multicolumn{2}{|l|}{ Apnoea frequency and type } \\
\hline Apnoea-hypopnoea index events $\cdot h^{-1}$ & $11.0(5.5-22.4)$ \\
\hline Central \% & $47.9(25.5-77.7)$ \\
\hline Mixed \% & $0.0(0.0-1.6)$ \\
\hline Obstructive \% & $47.4(21.4-74.4)$ \\
\hline \multicolumn{2}{|l|}{ Cardiometabolic disease occurrence and prevalence } \\
\hline Excessive erythrocytosis & 6 (3.2) \\
\hline Metabolic syndrome & $74(39.6)$ \\
\hline Diabetes & $8(4.3)$ \\
\hline Hypertension & $9(4.8)$ \\
\hline \multicolumn{2}{|l|}{ Cardiometabolic parameters } \\
\hline Haemoglobin $\mathrm{g} \cdot \mathrm{dL}^{-1}$ & $16.7(15.8-18.1)$ \\
\hline Haemoglobin A1c \% & $5.8(5.5-6.1)$ \\
\hline Fasting glucose $\mathrm{mg} \cdot \mathrm{dL}^{-1}$ & 89 (82-98) \\
\hline Fasting insulin $\mathrm{mIU} \cdot \mathrm{L}^{-1}$ & $6.9(4.1-12.0)$ \\
\hline Triglyceride $\mathrm{mg} \cdot \mathrm{dL}^{-1}$ & $134(107-194)$ \\
\hline High-density lipoprotein cholesterol $\mathrm{mg} \cdot \mathrm{dL}^{-1}$ & $39(34-47)$ \\
\hline Systolic blood pressure $\mathrm{mmHg}$ & $109(101-117)$ \\
\hline Diastolic blood pressure $\mathrm{mmHg}$ & $72(66-78)$ \\
\hline
\end{tabular}

Data are presented as $\mathrm{n}$, median (interquartile range) or $\mathrm{n}(\%)$. $\mathrm{SpO}_{2}$ : arterial oxygen saturation measured by pulse oximetry. 
to a reduction in mean nocturnal $\mathrm{SpO}_{2}$, their effect on oxygenation appear to be mitigated by more

frequent arousals (supplementary appendix S1). On average, men had greater haemoglobin concentrations than women $\left(18.0\right.$ versus $\left.15.8 \mathrm{~g} \cdot \mathrm{dL}^{-1} ; \mathrm{p}<0.001\right)$. Haemoglobin concentrations of older women were not significantly different from younger women (16.1 versus $15.7 ; \mathrm{p}=0.25$ ). The prevalence of excessive erythrocytosis was low in this sample. In contrast, metabolic syndrome was highly prevalent in Peruvian highlanders, although few had frank diabetes or hypertension.

\section{SDB and haemoglobin}

Single-variable associations between haemoglobin concentration and SDB are presented in figure 1. In men, haemoglobin concentration was inversely related to mean nocturnal $\mathrm{SpO}_{2}(\mathrm{p}<0.001)$ (figure 1a). In women aged $\geqslant 55$ years, who were likely to have undergone menopause, the haemoglobin concentration
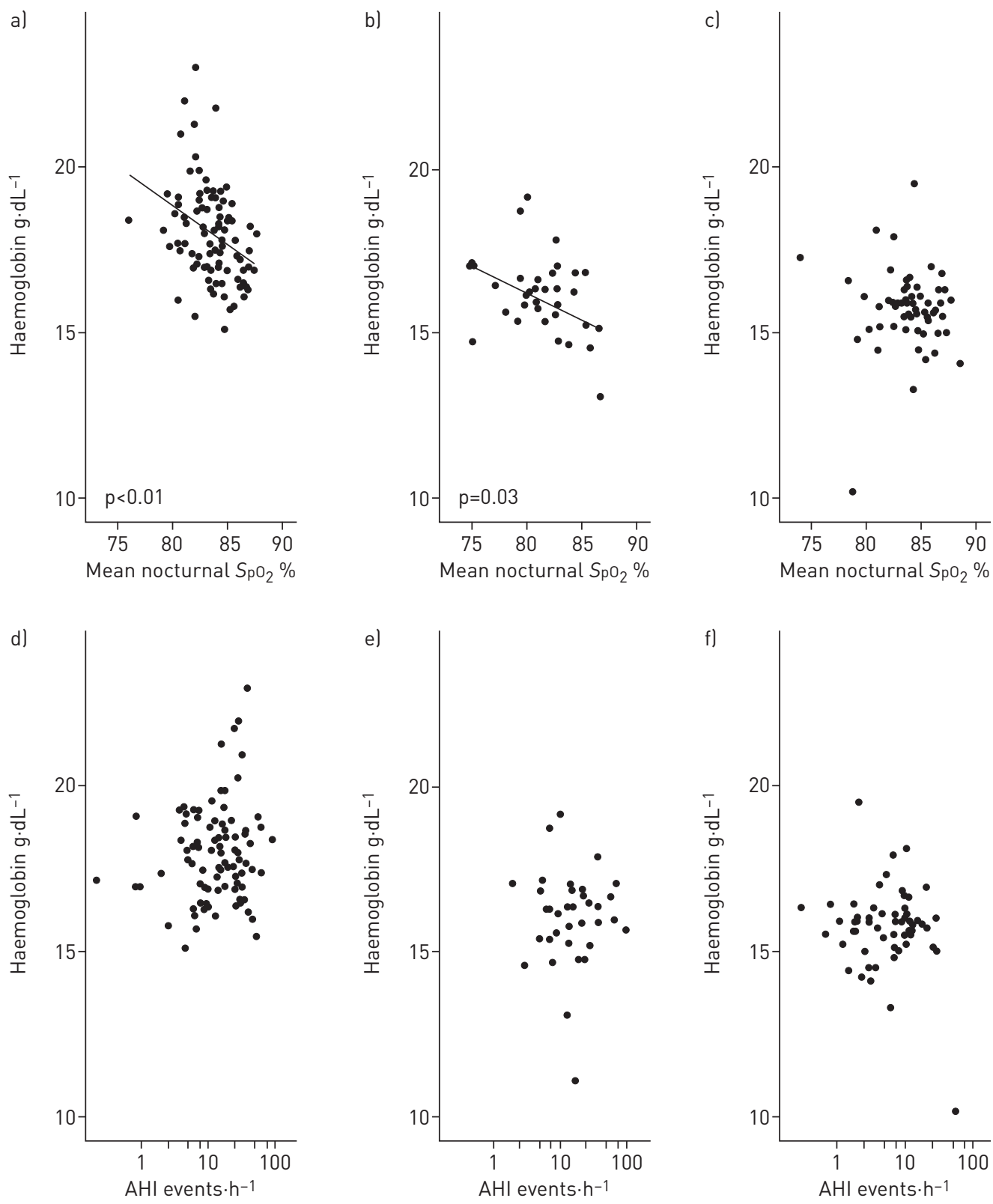

FIGURE 1 Haemoglobin versus sleep disordered breathing. $\mathrm{SpO}_{2}$ : arterial oxygen saturation measured by pulse oximetry; $\mathrm{AHI}$ : apnoea-hypopnoea index. Scatter plots of haemoglobin versus a-c) mean nocturnal $\mathrm{SpO}_{2}$ and $\mathrm{d}-$ f) $\mathrm{AHI}$ (semi-log scale) in $a, d$ ) men, b, e) women aged $\geqslant 55$ years and $c, f$ ) women aged $<55$ years. Solid lines represent fitted linear regression models in which a significant association was found. Haemoglobin was inversely associated with mean nocturnal $\mathrm{SpO}_{2}$ in men $(p<0.001)$ and women aged $\geqslant 55$ years $(p=0.03)$. AHI was not associated with haemoglobin concentration in men, women aged $\geqslant 55$ years or women aged $<55$ years. 
was also inversely associated with nocturnal $\mathrm{SpO}_{2}(\mathrm{p}=0.03)$ (figure 1b). In contrast, in women aged $<55$ years, haemoglobin was not associated with nocturnal $\mathrm{SpO}_{2}$ (figure 1c). Despite these associations with nocturnal $\mathrm{SpO}_{2}$, haemoglobin was not associated with $\mathrm{AHI}$ in either men or women (figure 1d-f).

Multivariable regression models also demonstrated that AHI was not associated with haemoglobin concentration in either men or in older or younger women after adjusting for $\mathrm{SpO}_{2}$ during wakefulness (figure 2a). Rather, nocturnal $\mathrm{SpO}_{2}$ residuals were inversely associated with haemoglobin concentration in men, but not women aged either $\geqslant 55$ or $<55$ years $(\mathrm{p}=0.02$ ) (figure $2 \mathrm{~b}$ ). After adjustment for age and $\mathrm{BMI}$, the $\mathrm{SpO}_{2}$ residual reductions remained significantly associated with haemoglobin concentration elevations in men, but not in older or younger women. Specifically, every $1 \%$ reduction in nocturnal $\mathrm{S}_{\mathrm{PO}_{2}}$ was associated with a $0.23 \mathrm{~g} \cdot \mathrm{dL}^{-1}$ elevation in haemoglobin concentration in men. Haemoglobin in older women was negatively associated with $\mathrm{SpO}_{2}$ during wakefulness $(\mathrm{p}<0.05)$ rather than sleep, although this association was not significant when adjusted for age and BMI (figure 2c).

\section{SDB and cardiometabolic outcomes}

Single-variable associations between SDB and glucose homeostasis are represented in figure 3 . Lower nocturnal $\mathrm{SpO}_{2}$ was associated with higher HbA1c $(\mathrm{p}<0.001)$, HOMA- $\beta(\mathrm{p}=0.04)$ and HOMA-IR $(\mathrm{p}=0.02)$, but not fasting glucose. Elevations in AHI were associated with elevations in $\mathrm{HbAlc}(\mathrm{p}<0.001)$ and HOMA-IR ( $p=0.02)$, but not HOMA- $\beta$. A trend toward increasing fasting glucose with AHI was also observed $(\mathrm{p}=0.07)$, consistent with the association between AHI and HbA1c.

In multivariable models, HbAlc and HOMA-IR elevations were both associated with AHI after adjusting for $\mathrm{SpO}_{2}$ during wakefulness ( $\mathrm{p}<0.001$ and $\mathrm{p}<0.05$, respectively) (figure $4 \mathrm{a}$ and $\mathrm{b}$ ). The association between $\mathrm{HbA1c}$ and AHI remained significant even after adjusting for age, sex and BMI $(\mathrm{p}<0.05)$, although the associations between HOMA-IR and AHI did not. The $\mathrm{SpO}_{2}$ residual was also inversely associated with HbAlc $(\mathrm{p}<0.05)$ (figure $4 \mathrm{~d})$, suggesting a link between nocturnal desaturation and glucose intolerance. This association was not significant after adjusting for age, sex and BMI. Nonetheless, HbA1c elevations were also associated with reductions in $\mathrm{SpO}_{2}$ during wakefulness. Of note, the inclusion of nocturnal $\mathrm{S}_{2} \mathrm{O}_{2}$
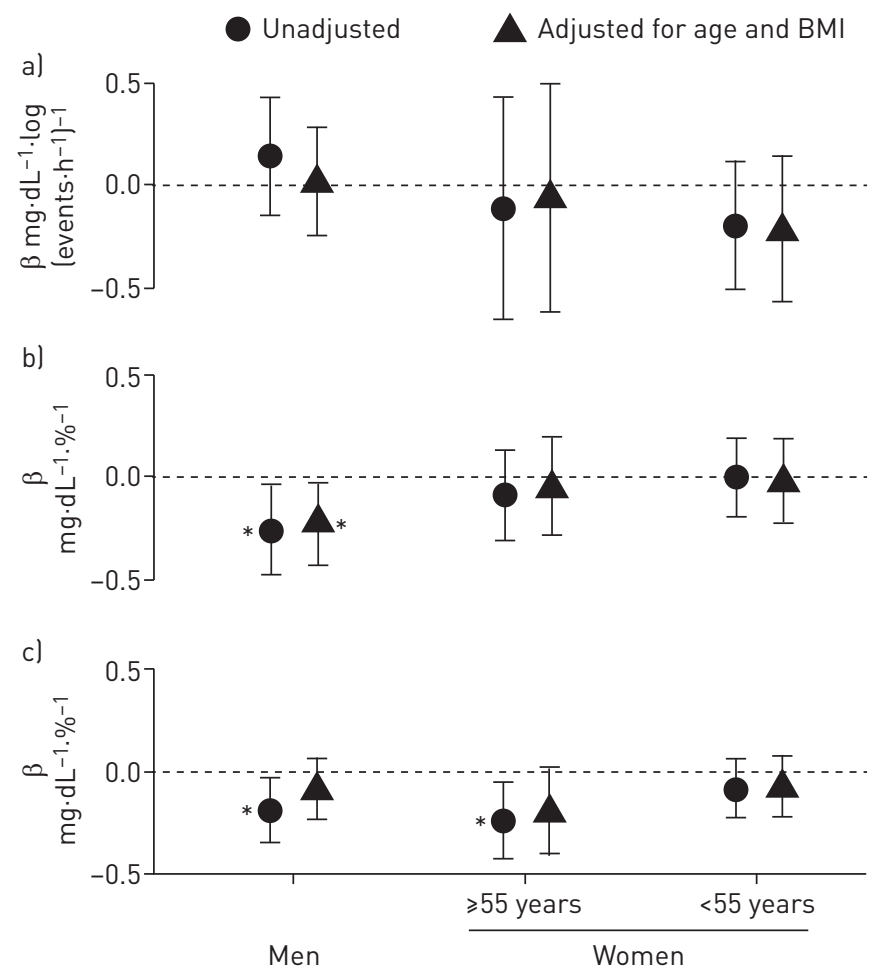

FIGURE 2 Multivariable models of haemoglobin as a function of sleep disordered breathing components. $\mathrm{SpO}_{2}$ : arterial oxygen saturation measured by pulse oximetry; $\mathrm{AHI}$ : apnoea-hypopnoea index. The results of multivariable models ( $\beta(95 \% \mathrm{CI})$ to examine the association between a) haemoglobin and AHI (log AHI), b) the degree to which nocturnal hypoxaemia was attenuated $\left(\mathrm{SpO}_{2}\right.$ residual), and $\mathrm{c}$ ) oxygenation during wakefulness (wake $\mathrm{SpO}_{2}$ ) in men, women aged $\geqslant 55$ years and women aged $<55$ years. *: $p<0.05$. These models demonstrated that $\mathrm{AHI}$ was not associated with haemoglobin. Reductions in $\mathrm{SpO}_{2}$ residual (lower than expected nocturnal $\mathrm{SpO}_{2}$ ) independently predicted haemoglobin elevations in men. Wake $\mathrm{SpO}_{2}$ predicted haemoglobin in women aged $\geqslant 55$ years in unadjusted but not adjusted models. 

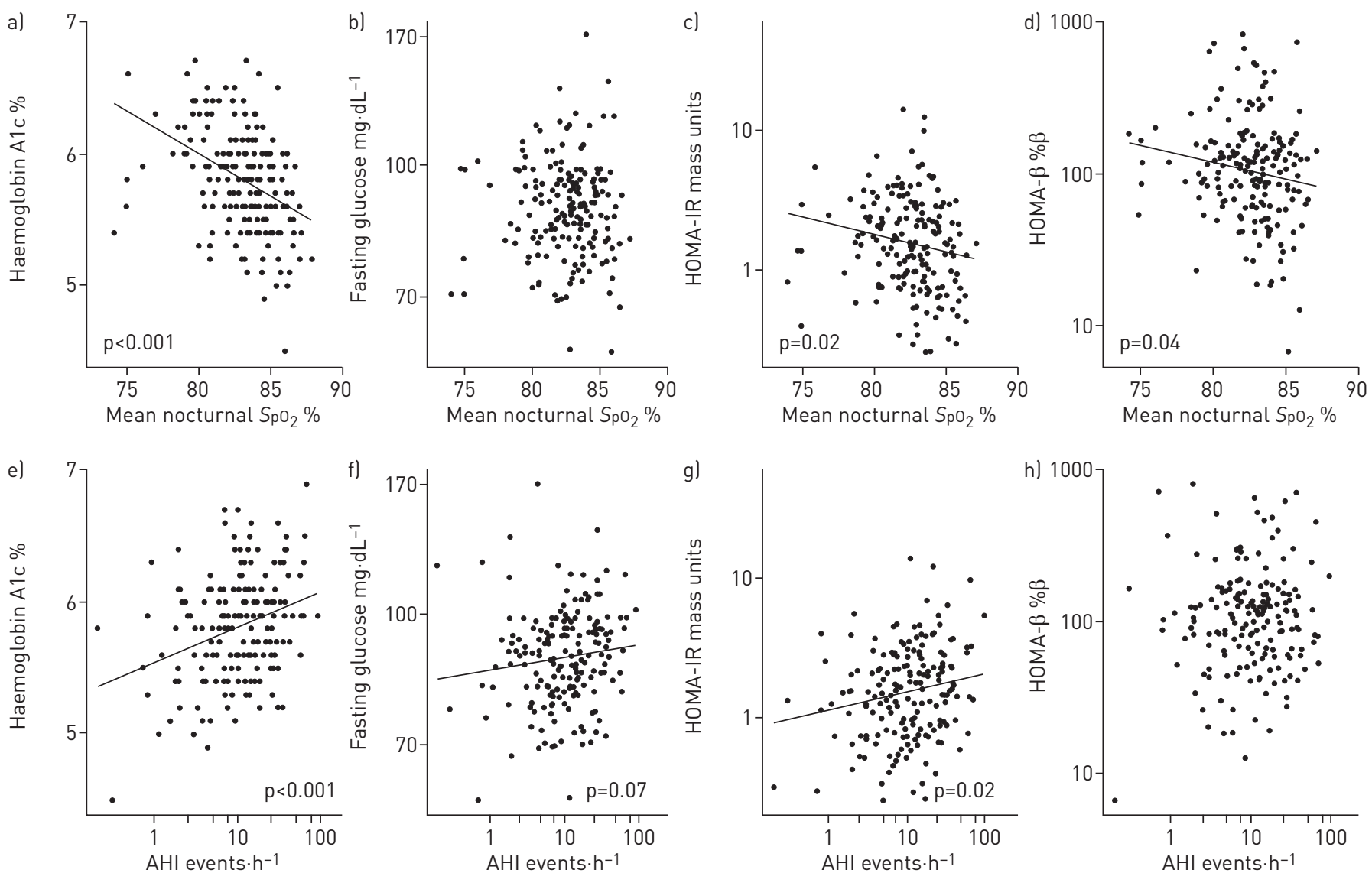

FIGURE 3 Glucose homeostasis parameters versus sleep disordered breathing. HOMA-IR: homeostatic model-based assessment of insulin resistance; HOMA- $\beta$ : homeostatic model-based assessment of $\beta$-cell function; $\mathrm{SpO}_{2}$ : arterial oxygen saturation measured by pulse oximetry; AHI: apnoea-hypopnoea index. a-h) Scatter plots of a, e) haemoglobin A1c, b, f) fasting glucose, c, g) HOMA-IR and d, h) HOMA- $\beta$ plotted as functions of $a-d$ ) mean nocturnal $\mathrm{SpO}_{2}$ and $e-h$ ) AHI (semi-log scale). The $y$-axes for fasting glucose, HOMA-IR and HOMA- $\beta$ have been transformed to reflect the transformations used in linear regression. Solid lines represent fitted linear regression models in which a significant association was found.

residual and AHI in the model did not diminish the strength or significance of the association between $\mathrm{HbA1c}$ and $\mathrm{SpO}_{2}$ during wakefulness (supplemental appendix S7).

To account for potential confounding by haemoglobin concentration on HbAlc, we adjusted for this parameter and found that it did not alter the above associations between SDB and HbAlc (supplementary appendix S2). Sensitivity analyses also demonstrated that the association between HbA1c and AHI was not significantly altered even when subjects with diabetes were included or when waist circumference and waist-to-hip ratio were substituted for BMI in multivariable models (supplementary appendices S3 and S4).

In single-variable models of the remaining cardiometabolic outcomes, AHI was associated with systolic blood pressure $(\mathrm{p}<0.001)$ and triglycerides $(\mathrm{p}<0.01)$, but not high-density lipoprotein (HDL) cholesterol (supplementary appendix S5). A borderline association between AHI and diastolic blood pressure was also demonstrated $(\mathrm{p}=0.05)$. Mean nocturnal $\mathrm{SpO}_{2}$ was associated with triglycerides $(\mathrm{p}<0.001)$, but not blood pressure or cholesterol. After adjusting for $\mathrm{SpO}_{2}$ during wakefulness, multivariable models of SDB demonstrated that AHI was associated with systolic blood pressure $(\mathrm{p}=0.01)$ and serum triglyceride levels $(\mathrm{p}<0.01)$, but not diastolic blood pressure or HDL cholesterol. These associations were not significant after adjusting for age, sex and BMI.

\section{Discussion}

We demonstrated that nocturnal hypoxaemia and sleep apnoea were associated with distinct patterns of cardiometabolic dysfunction in a large sample of native highlanders. Specifically, increasing degrees of nocturnal hypoxaemia rather than AHI predicted elevations in haemoglobin concentrations in male highlanders independent of age and BMI, predisposing them to CMS [33]. A similar association was observed in older women, which was primarily related to hypoxaemia during wakefulness rather than during sleep. Conversely, AHI rather than severity of nocturnal hypoxaemia was associated with metabolic 

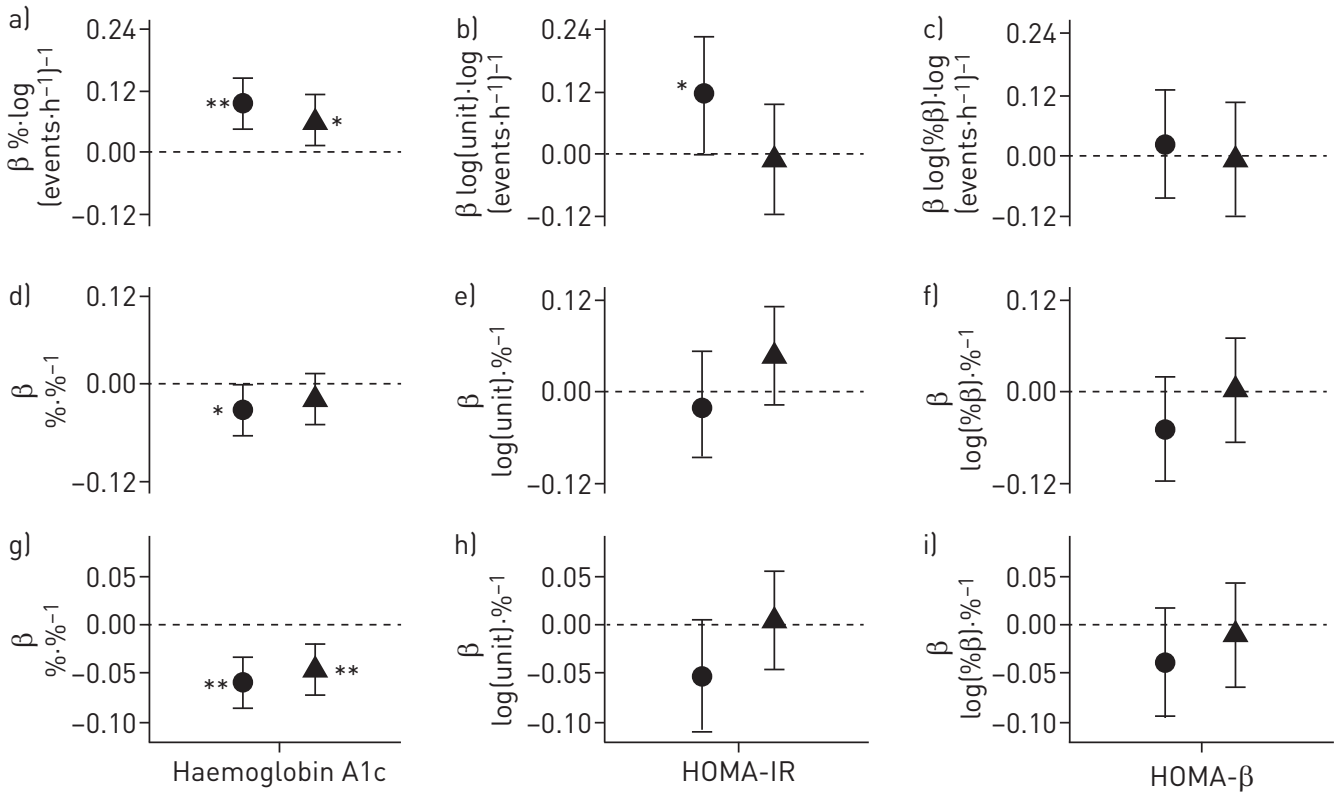

FIGURE 4 Multivariable models of glucose homeostasis as functions of sleep disordered breathing components. $\mathrm{SpO}_{2}$ : arterial oxygen saturation measured by pulse oximetry; $\mathrm{AHI}$ : apnoea-hypopnoea index; HbA1c: haemoglobin A1c; HOMA-IR: homeostatic model-based assessment of insulin resistance; HOMA- $\beta$ : homeostatic model-based assessment of $\beta$-cell function; BMI: body mass index. a-i) Results of multivariable models examining associations $(\beta(95 \% \mathrm{CI}))$ between glucose homeostasis markers (HbA1c, HOMA-IR and HOMA- $\beta$ ) and a-c) AHI (log AHI), $d-f)$ the degree to which nocturnal hypoxaemia was attenuated $\left(\mathrm{SpO}_{2}\right.$ residual) and $\mathrm{g}-\mathrm{i}$ ) oxygenation during wakefulness (wake $\mathrm{SpO}_{2}$ ) are presented. ${ }^{*}: \mathrm{p}<0.05$; ${ }^{* *}$ : $\mathrm{p}<0.001$. AHI was independently associated with $\mathrm{HbA} 1 \mathrm{c}$.

dysregulation. Specifically, AHI was associated with HbA1c elevations, independent of age, BMI, sex, sleep and wake levels of $\mathrm{SpO}_{2}$, and HOMA-IR elevations, independent of $\mathrm{SpO}_{2}$ during sleep and wakefulness. Taken together, our findings suggest that recurrent SDB episodes with intermittent hypoxaemia contributed to glucose intolerance, whereas sustained nocturnal hypoxaemia contributed to elevations in haemoglobin, a key marker for CMS in highlanders. As discussed later in this section, our findings also suggest that differential disturbances in sleep and gas exchange threaten highland communities with excess metabolic and cardiovascular morbidity and mortality, respectively.

Our study demonstrated a novel association between nocturnal oxygenation and haemoglobin concentration in male highlanders. Of note, this association was independent of age, BMI and $\mathrm{SpO}_{2}$ during wakefulness. These findings in Andean highlanders strengthen the link between sustained hypoxaemia and elevated haemoglobin, a key biomarker for CMS susceptibility in highlanders [33]. Investigators have demonstrated similar findings in observational case series of CMS highland patients compared with highland controls [31, 34-36]. Nonetheless, previous population-based studies of highlanders have not confirmed this association, possibly due to small sample sizes or less extreme exposure to altitude [37]. In contrast, our sample size was considerably larger, which allowed us to examine associations between several SDB components (i.e. AHI and $\mathrm{SpO}_{2}$ residual) and haemoglobin concentration while adjusting for daytime hypoxaemia. Although apnoeic episodes were associated with worsening nocturnal hypoxaemia, we still found no association between AHI and haemoglobin concentration in single or multivariable models, indicating that sustained rather than intermittent hypoxaemia accounts for haemoglobin elevations in this population. Among highland populations worldwide, Andean highlanders may be particularly susceptible to the effects of chronic hypoxia on haemoglobin concentration [38-40], although the prevalence of excessive erythrocytosis remained relatively low. Several factors may have contributed to the development of this condition in highlanders including in addition to genetic background, degree of ambient hypoxia, vital capacity, age, obesity and comorbid conditions [41]. Our study extends prior work by highlighting the potential impact of nocturnal desaturation as a risk factor for elevated haemoglobin concentration in highlanders.

In contrast to nocturnal hypoxaemia, we found that recurrent SDB episodes (AHI) were associated with elevations in $\mathrm{HbA} 1 \mathrm{c}$ in age-, weight-, sex- and $\mathrm{S}_{\mathrm{PO}_{2}}$-adjusted models, and with HOMA-IR, even after adjusting for $\mathrm{SpO}_{2}$ during sleep and wakefulness. These findings in highlanders are consistent with recognised associations between glucose intolerance and sleep apnoea in lowlanders [20]. In lowlanders, glucose 
intolerance in sleep apnoea is attributed to intermittent hypoxaemia and recurrent arousals from sleep, and improves with continuous positive airway pressure intervention [24, 42, 43]. Our findings provide further insight into mechanisms of glucose dysregulation in SDB by dissecting associations with sustained hypoxaemia and recurrent SDB episodes along with intermittent hypoxaemia and repetitive arousals from sleep. Our findings suggest that the frequency of events rather than the severity of nocturnal hypoxaemia could account for glucose intolerance in these highlanders. In addition to recurrent SDB episodes, we confirmed our previous finding that sustained hypoxaemia during wakefulness was also associated with HbA1c elevations (figure $4 \mathrm{a}, \mathrm{d}$ and $\mathrm{g}$ ) [27]. The present study extends this previous finding by demonstrating an association between glucose intolerance and $\mathrm{AHI}$, independent of daytime $\mathrm{SpO}_{2}$. Moreover, adjusting for $\mathrm{AHI}$ in this model did not attenuate the association between $\mathrm{SpO}_{2}$ during wakefulness and $\mathrm{HbA1c}$ (supplementary appendix S7), suggesting that both apnoeic episodes and $\mathrm{SpO}_{2}$ account for $\mathrm{HbA1c}$ elevations in highlanders. Thus, our findings in Peruvian highlanders suggest that daytime sustained hypoxaemia and nocturnal recurrent SDB episodes both contribute to the severity of glucose dysregulation in highlanders.

Our study has several limitations worth considering when interpreting our findings. First, the cross-sectional design in a convenience sample precludes drawing causal inferences between cardiometabolic dysfunction and SDB. We recognise that unmeasured confounders and sampling bias could influence the association between SDB and cardiometabolic dysfunction. Nevertheless, our findings are consistent with biologically plausible mechanisms and previous studies, which have linked SDB to CMS and glucose intolerance. Furthermore, our main findings were of sufficient magnitude and remained significant after adjusting for age, sex and BMI. In addition, sensitivity analyses (supplementary appendix S4) demonstrated alternative measures of obesity, including waist circumference and waist-to-hip ratio, did not attenuate the association between HbAlc and AHI. Second, the overall prevalence of chronic diseases (i.e. excessive erythrocytosis and diabetes) in our cohort was low, which prevented us from examining the association between SDB and chronic disease. Nevertheless, the observed associations of haemoglobin and HbAlc with nocturnal hypoxaemia and AHI, respectively, suggest that these nocturnal exposures impose cardiometabolic stress in highlanders, placing highlanders at risk for diabetes and CMS. Moreover, the relative low prevalence of these chronic conditions may have allowed us to detect associations between the markers of metabolic stress and SDB in a relatively small sample size. Third, polygraphy was employed to assess SDB rather than polysomnography. Nevertheless, our methods incorporated a validated algorithm for actigraphy to estimate sleep-wake state and movement arousals [29], possibly leading to an overestimation of sleep time and corresponding underestimation of sleep disturbances and SDB severity, as measured by SDB event frequency and wake-to-sleep reductions in $\mathrm{SpO}_{2}$. Fourth, hypoxia generally elevates haemoglobin concentrations, which can lower HbA1c and the likelihood of detecting significant associations. Nevertheless, inclusion of haemoglobin to account for the potential effects of haemoglobin elevations in sensitivity analyses did not significantly change our findings. Fifth, we recognise that it is difficult to separate the effects of sleep apnoea from nocturnal hypoxaemia on outcomes, since sleep apnoea is frequently associated with greater degrees of nocturnal hypoxaemia. Nonetheless, we dissected the nocturnal component $\left(\mathrm{SpO}_{2}\right.$ residual) from overall levels of oxygenation and found that it predicted haemoglobin independent of oxygenation during wakefulness. A similar approach allowed us to isolate an association between $\mathrm{HbAlc}$ and $\mathrm{AHI}$, independent of nocturnal desaturation $\left(\mathrm{SpO}_{2}\right.$ residual). Sixth, we recognise that biomarkers, including $\mathrm{HbAlc}$ and fasting glucose, can be confounded by diabetes and its treatment. Nevertheless, sensitivity analyses demonstrated that the association between AHI and HbA1c did not significantly change with the inclusion of diabetic study subjects. Seventh, we acknowledge our study was limited to only one highland group (i.e. Andeans). Highland populations are known to have adopted different strategies for meeting the challenges of living at altitude, which could impact links between cardiometabolic dysfunction and SDB. Residual confounding from genetic adaptations and environmental exposures in specific highland groups (based on differences in food, water and rural/urban exposures) may also have influenced manifestations of both SDB and related cardiometabolic outcomes [44].

Our findings are consistent with the notion that nocturnal hypoxaemia and recurrent apnoeic events could exert differential effects on cardiometabolic outcomes. In our study, highlanders demonstrated specific SDB patterns characterised by alterations in both oxygenation and sleep to varying degrees (figure 5). When sleep continuity was maintained, highlanders were exposed to prolonged nocturnal periods of sustained rather than intermittent periods of hypoxaemia. This pattern was associated with elevated haemoglobin, a hallmark of CMS [33]. In contrast, highlanders who aroused readily from SDB episodes appeared to experience a greater frequency of SDB episodes with intermittent rather than sustained hypoxaemia. Under these circumstances, arousals from sleep can mitigate hypoxaemia severity. Our findings suggest that periodic SDB episodes with attendant arousals and intermittent desaturations can aggravate glucose homeostasis but do not confer risk of excessive erythrocytosis and CMS. These concepts lead us to speculate that alleviating nocturnal hypoxaemia and sleep apnoea in highlanders could improve polycythaemia and glucose intolerance, respectively. We further speculate that lowland patients with underlying cardiopulmonary 


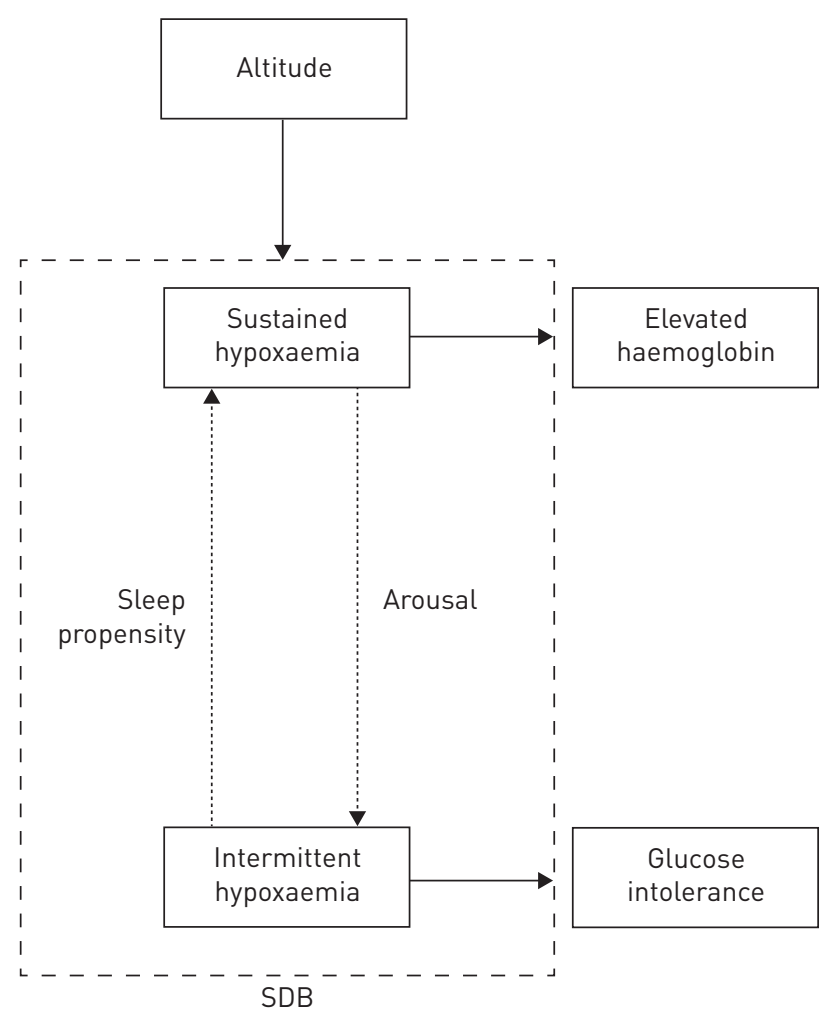

FIGURE 5 Cardiometabolic correlates of specific sleep disordered breathing (SDB) patterns. High altitude causes hypoxaemia and predisposes to SDB. SDB patterns and cardiometabolic outcomes may be determined nocturnal responses to hypoxaemia. A high sleep propensity may maintain sleep continuity, but can result in prolonged apnoeic events and sustained nocturnal hypoxaemia, which was associated with elevated haemoglobin. Alternatively, arousals from sleep can mitigate nocturnal hypoxaemia, but result in apnoeas and hypopnoeas with intermittent hypoxaemia, which was associated with glucose intolerance.

disease, who often demonstrate similar degrees of nocturnal hypoxaemia and sleep apnoea, remain at increased risk for cardiometabolic dysfunction and accelerated disease progression. Thus, sleep remains an untapped therapeutic window during which cardiometabolic risk can be mitigated. Further mechanistic studies are required to establish causal mechanisms linking SDB with the development of cardiometabolic diseases and develop nocturnal therapeutic strategies.

\section{Acknowledgements}

We would like to acknowledge Francis Sgambati and the Center for Interdisciplinary Sleep Research and Education (CISRE) at the Johns Hopkins School of Medicine (Baltimore, MD, USA) for their support in data transfer and management. We would also like to thank ResMed Ltd (Bella Vista, New South Wales, Australia) for their generous donation of ApneaLink Plus recording devices.

Author contributions: Study design: A.R. Schwartz, W. Checkley, L.V. Pham. Data acquisition: R. Arias, N.G. Schwartz, A. Rattner. Data analysis: A.R. Schwartz, L.V. Pham, N.G. Schwartz, R.S. Arias. Data interpretation: A.R. Schwartz, W. Checkley, R.H. Gilman, J.J. Miranda, V.Y. Polotsky, N.G. Schwartz, C.H. Miele, L.V. Pham. Drafting of manuscript: A.R. Schwartz, L.V. Pham. Critical revision: all authors.

\section{References}

1 Arai Y, Tatsumi K, Sherpa NK, et al. Impaired oxygenation during sleep at high altitude in Sherpa. Respir Physiol Neurobiol 2002; 133: 131-138.

2 Nocturnal Oxygen Therapy Trial Group. Continuous or nocturnal oxygen therapy in hypoxemic chronic obstructive lung disease: a clinical trial. Ann Intern Med 1980; 93: 391-398.

3 Górecka D, Gorzelak K, Sliwiński P, et al. Effect of long-term oxygen therapy on survival in patients with chronic obstructive pulmonary disease with moderate hypoxaemia. Thorax 1997; 52: 674-679.

4 Chaouat A, Weitzenblum E, Kessler $\mathrm{R}$, et al. A randomized trial of nocturnal oxygen therapy in chronic obstructive pulmonary disease patients. Eur Respir J 1999; 14: 1002-1008.

5 Fletcher EC, Luckett RA, Goodnight-White S, et al. A double-blind trial of nocturnal supplemental oxygen for sleep desaturation in patients with chronic obstructive pulmonary disease and a daytime $\mathrm{PaO}_{2}$ above $60 \mathrm{~mm} \mathrm{Hg}$. Am Rev Respir Dis 1992; 145: 1070-1076.

6 Flick MR, Block AJ. Continuous in-vivo monitoring of arterial oxygenation in chronic obstructive lung disease. Ann Intern Med 1977; 86: 725-730. 
7 Coccagna G, Mantovani M, Brignani F, et al. Continuous recording of the pulmonary and systemic arterial pressure during sleep in syndromes of hypersomnia with periodic breathing. Bull Physiopathol Respir 1972; 8: $1159-1172$

8 Motta J, Guilleminault C, Schroeder JS, et al. Tracheostomy and hemodynamic changes in sleep-inducing apnea. Ann Intern Med 1978; 89: 454-458.

9 Bickelmann AG, Burwell CS, Robin ED, et al. Extreme obesity associated with alveolar hypoventilation: a Pickwickian syndrome. Am J Med 1956; 21: 811-818.

10 Monge C. Chronic mountain sickness. Physiol Rev 1943; 23: 166-183.

11 Monge CC, Whittembury J. Chronic mountain sickness. Johns Hopkins Med J 1976; 139: Suppl., 87-89.

12 Monge-C C, Arregui A, León-Velarde F. Pathophysiology and epidemiology of chronic mountain sickness. Int $J$ Sports Med 1992; 13: Suppl. 1, S79-S81.

13 Marin JM, Carrizo SJ, Vicente E, et al. Long-term cardiovascular outcomes in men with obstructive sleep apnoeahypopnoea with or without treatment with continuous positive airway pressure: an observational study. Lancet 2005; 365: 1046-1053.

14 Punjabi NM, Caffo BS, Goodwin JL, et al. Sleep-disordered breathing and mortality: a prospective cohort study. PLoS Med 2009; 6: e1000132.

15 Gottlieb DJ, Yenokyan G, Newman AB, et al. Prospective study of obstructive sleep apnea and incident coronary heart disease and heart failure: the Sleep Heart Health Study. Circulation 2010; 122: 352-360.

16 Nieto F, Young TB, Lind BK, et al. Association of sleep-disordered breathing, sleep apnea, and hypertension in a large community-based study. JAMA 2000; 283: 1829-1836.

17 Shahar E, Whitney CW, Redline S, et al. Sleep-disordered breathing and cardiovascular disease: cross-sectional results of the Sleep Heart Health Study. Am J Respir Crit Care Med 2001; 163: 19-25.

18 Peppard PE, Young T, Palta M, et al. Prospective study of the association between sleep-disordered breathing and hypertension. N Engl J Med 2000; 342: 1378-1384.

19 Young T, Finn L, Peppard PE, et al. Sleep disordered breathing and mortality: eighteen-year follow-up of the Wisconsin Sleep Cohort. Sleep 2008; 31: 1071-1078.

20 Resnick HE, Redline S, Shahar E, et al. Diabetes and sleep disturbances: findings from the Sleep Heart Health Study. Diabetes Care 2003; 26: 702-709.

21 Bakker JP, Weng J, Wang R, et al. Associations between obstructive sleep apnea, sleep duration, and abnormal fasting glucose. The Multi-Ethnic Study of Atherosclerosis. Am J Respir Crit Care Med 2015; 192: 745-753.

22 Dorey-Stein Z, Amorosa VK, Kostman JR, et al. Severe weight gain, lipodystrophy, dyslipidemia, and obstructive sleep apnea in a human immunodeficiency virus-infected patient following highly active antiretroviral therapy. $J$ Cardiometab Syndr 2008; 3: 111-114.

23 Polotsky VY, Patil SP, Savransky V, et al. Obstructive sleep apnea, insulin resistance, and steatohepatitis in severe obesity. Am J Respir Crit Care Med 2009; 179: 228-234.

24 Pamidi S, Wroblewski K, Stepien M, et al. Eight hours of nightly continuous positive airway pressure treatment of obstructive sleep apnea improves glucose metabolism in patients with prediabetes. a randomized controlled trial. Am J Respir Crit Care Med 2015; 192: 96-105.

25 Gottlieb DJ, Punjabi NM, Mehra R, et al. CPAP versus oxygen in obstructive sleep apnea. N Engl J Med 2014 370: 2276-2285.

26 Schwartz NG, Rattner A, Schwartz AR, et al. Sleep disordered breathing in four resource-limited settings in Peru: prevalence, risk factors, and association with chronic diseases. Sleep 2015; 38: 1451-1459.

27 Miele $\mathrm{CH}$, Schwartz AR, Gilman $\mathrm{RH}$, et al. Increased cardiometabolic risk and worsening hypoxemia at high altitude. High Alt Med Biol 2016; 17: 93-100.

28 Miranda JJ, Bernabe-Ortiz A, Smeeth L, et al. Addressing geographical variation in the progression of non-communicable diseases in Peru: the CRONICAS cohort study protocol. BMJ Open 2012; 2: e000610.

29 Kushida CA, Chang A, Gadkary C, et al. Comparison of actigraphic, polysomnographic, and subjective assessment of sleep parameters in sleep-disordered patients. Sleep Med 2001; 2: 389-396.

30 Berry RB, Budhiraja R, Gottlieb DJ, et al. Rules for scoring respiratory events in sleep: update of the 2007 AASM Manual for the Scoring of Sleep and Associated Events. J Clin Sleep Med 2012; 8: 597-619.

31 Julian CG, Vargas E, Gonzales M, et al. Sleep-disordered breathing and oxidative stress in preclinical chronic mountain sickness (excessive erythrocytosis). Respir Physiol Neurobiol 2013; 186: 188-196.

32 Matthews DR, Hosker JP, Rudenski AS, et al. Homeostasis model assessment: insulin resistance and beta-cell function from fasting plasma glucose and insulin concentrations in man. Diabetologia 1985; 28: 412-419.

33 León-Velarde F, Maggiorini M, Reeves JT, et al. Consensus statement on chronic and subacute high altitude diseases. High Alt Med Biol 2005; 6: 147-157.

34 Kryger M, Glas R, Jackson D, et al. Impaired oxygenation during sleep in excessive polycythemia of high altitude: improvement with respiratory stimulation. Sleep 1978; 1: 3-17.

35 Spicuzza L, Casiraghi N, Gamboa A, et al. Sleep-related hypoxaemia and excessive erythrocytosis in Andean high-altitude natives. Eur Respir J 2004; 23: 41-46.

36 Sun S, Oliver-Pickett C, Ping Y, et al. Breathing and brain blood flow during sleep in patients with chronic mountain sickness. J Appl Physiol 1996; 81: 611-618.

37 Bouscoulet LT, Vázquez-García JC, Muiño A, et al. Prevalence of sleep related symptoms in four Latin American cities. J Clin Sleep Med 2008; 4: 579-585.

38 Beall CM, Brittenham GM, Strohl KP, et al. Hemoglobin concentration of high-altitude Tibetans and Bolivian Aymara. Am J Phys Anthropol 1998; 106: 385-400.

39 Winslow RM, Chapman KW, Gibson CC, et al. Different hematologic responses to hypoxia in Sherpas and Quechua Indians. J Appl Physiol 1989; 66: 1561-1569.

40 Beall CM. Two routes to functional adaptation: Tibetan and Andean high-altitude natives. Proc Natl Acad Sci USA 2007; 104: 8655-8660.

41 De Ferrari A, Miranda JJ, Gilman RH, et al. Prevalence, clinical profile, iron status, and subject-specific traits for excessive erythrocytosis in Andean adults living permanently at 3,825 meters above sea level. Chest 2014; 146: 1327-1336. 
42 Louis M, Punjabi NM. Effects of acute intermittent hypoxia on glucose metabolism in awake healthy volunteers. J Appl Physiol 2009; 106: 1538-1544.

43 Stamatakis KA, Punjabi NM. Effects of sleep fragmentation on glucose metabolism in normal subjects. Chest 2010; 137: 95-101.

44 Beall CM. Human adaptability studies at high altitude: research designs and major concepts during fifty years of discovery. Am J Hum Biol 2013; 25: 141-147. 